\title{
AN ELECTRON TUBE AMPLIFIER FOR AMPLIFYING DIRECT CURRENT
}

BY

H. A. SNow

In various fields in electrical work, particularly in radio work, it is important to have a relay possessing negligible time lag, capable of operating on small impressed currents, of the order of 10 milliamperes. Such a device may be found useful, for example, in recorders for registering telegraphic or radio signals, in apparatus for remote electrical control, in railway signaling work, and for operating an oscillograph from a source of very small current.

The following paper describes a resistance coupled electron tube amplifier designed to amplify a direct or alternating current of 10 to 20 milliamperes to 110 to 200 milliamperes to take the place of a special type of polarized relay at present used for this purpose. The conditions under which the relay operates and which are imposed on the amplifier follow:

\section{Conditions}

The current operating the relay is either an alternating current of 10 to 20 milliamperes at a frequency of about 40 cycles or a direct current of the same magnitude in either direction, both of which are applied intermittently from a high impedance source. The input impedance of the relay is about $750 \mathrm{ohms}$ with an allowable increase to $1000 \mathrm{ohms}$. The impedance of the output circuit is 50 to $100 \mathrm{ohms}$. When a direct current in a given direction is supplied to the input of the amplifier, an amplified direct current will be produced in the output circuit. When a direct current in the opposite direction is supplied to the input, an amplified direct current in the opposite direction will be produced in the output circuit. The amplifier will amplify alternating currents of either low commercial frequencies or high radio frequencies with very little distortion.

\section{TuBes}

The electron tubes selected for the amplifier tests were UV-202 Radiotron 5 watt power tubes, because of their high filament emission and steadiness of operation with a high plate current. 


\section{Circuits}

Since the electron tube amplifier is essentially a voltage operated device of high impedance, it is not readily adapted for efficient current amplification. For highest efficiency the highest possible voltage should be produced from the input current it is desired to amplify. The type of amplifier circuit that may be used is limited by the fact that the input consists partially of direct current, to a resistance coupled circuit employing the voltage drop across a resistance for the input to each stage, so the maximum voltage available with 10 milliamperes flowing in the input circuit is the drop across a resistance in the input circuit. The resistance being limited to $1000 \mathrm{ohms}$, the maximum voltage available is 10 volts. This requires more than one stage of amplification for efficient use of the tubes.
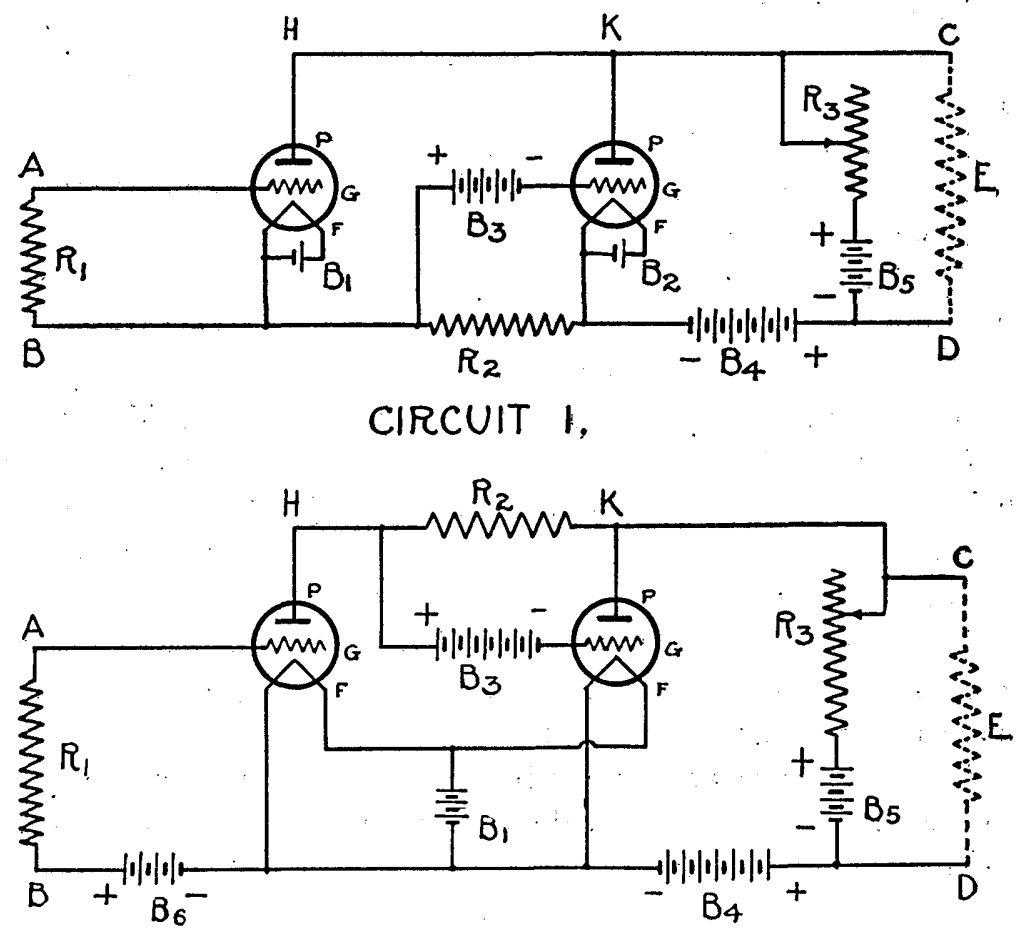

CIRCUIT 2,

Fig. 1-Amplifier circuits 


\section{Circuit Description}

Two circuits each having two resistance-coupled stages were set up and adjusted for maximum current amplification. These circuits are shown diagrammatically in Fig. 1.

In both circuits 1 and 2 of Fig. 1:

$A$ and $B$ are the input terminals.

$C$ and $D$ are the output terminals.

$E$ (in dotted lines) represents the impedance of the output circuit. In these tests $E$ was a resistance of 50 ohms.

$F, G$, and $P$ are respectively the filaments, grids and plates of the tubes.

$\therefore H=$ First stage.

$K=$ Second stage. For simplicity only one tube is shown in each stage, although several connected in parallel were used.

$B_{1}$ and $B_{2}=8$ volt batteries of sufficient capacity to supply the filaments connected to each battery. Each tube requires 2.3 amperes.

$B_{3}=$ Source of grid voltage of 22 to 110 volts, according to tubes used, capable of carrying about 40 milliamperes in a direction opposite to the voltage of this source.

$B_{4}=$ Source of plate voltage, 220 volts, capable of delivering 0.5 amperes.

$B_{5}=40$ volt battery to supply 200 milliamperes.

$B_{6}=$ (circuit 2 only) 12 volt battery to supply 20 milliamperes.

$R_{1}=$ Input resistance of $1000 \mathrm{ohms}$. The sensitivity of the amplifier can be controlled completely by varying this resistance. Increasing the resistance increases the current amplification and reducing the resistance decreases the amplification.

$R_{2}=$ Coupling resistance of $10000 \mathrm{ohms}$ to $55000 \mathrm{ohms}$, according to number of tubes used. Both $R_{1}$ and $R_{2}$ should have a current capacity of 30 milliamperes.

$R_{3}=$ Resistance which must be variable by small steps from 150 to $300 \mathrm{ohms}$, such as a slide wire rheostat. Current carrying capacity 200 milliamperes.

\section{TEsts}

With the two circuits shown in Fig. 1 adjusted to give the highest amplification, measurements were made of the output current 
for an input voltage of 10 volts positive and negative which corresponds to an input current of 10 milliamperes through the input resistance of $1000 \mathrm{ohms}$. The current amplification, that is, the ratio of the output to the input current, is obtained by dividing the measured output current by the 10 milliampere input current.

\section{Results}

The following table shows the amplification obtained with both circuits, using the tubes as indicated in the first three columns and also gives the values of coupling resistance and grid voltage $\left(R_{2}\right.$ and $B_{3}$ respectively of Fig. 1) required for best operation in each case:

TABLE 1

Circuit 1

\begin{tabular}{|c|c|c|c|c|c|c|}
\hline \multicolumn{3}{|c|}{ Number of Tubes } & \multirow{2}{*}{$\mathrm{R}_{2} \mathrm{Ohms}$} & \multirow{2}{*}{ Ba Volts } & \multirow{2}{*}{$\begin{array}{l}\text { Output Current } \\
\text { with } 10 \text { ma input }\end{array}$} & \multirow{2}{*}{$\begin{array}{c}\text { Current } \\
\text { Amplification }\end{array}$} \\
\hline Total No. & First Stage & Second Stage & & & & \\
\hline $2^{1}$ & 1 & 1 & 46000 & 100 & 60 & 6 \\
\hline $3^{1}$ & 1 & 2 & 46000 & 100 & 100 & 10 \\
\hline 4 & 1 & 3 & 56000 & 88 & 120 & 12 \\
\hline 5 & 2 & 3 & 14000 & 110 & 130 & 13 \\
\hline 6 & 2 & 4 & 14000 & 110 & 160 & 16 \\
\hline
\end{tabular}

TABLE 1

Circuit 2

\begin{tabular}{|c|c|c|c|c|c|c|}
\hline \multicolumn{3}{|c|}{ Number of Tubes } & \multirow[b]{2}{*}{$R_{2}$ ohms } & \multirow[b]{2}{*}{$\mathrm{B}_{\mathfrak{z}}$ voltis } & \multirow{2}{*}{$\begin{array}{l}\text { Output Current } \\
\text { with } 10 \mathrm{ma} \\
\text { input }\end{array}$} & \multirow{2}{*}{$\begin{array}{c}\text { Current } \\
\text { Amplification }\end{array}$} \\
\hline Total No. & First Stage & Second Stage & & & & \\
\hline $3^{1}$ & 1 & 2 & 12000 & 45 & 70 & 7 \\
\hline $4^{1}$ & 2 & 2 & 8000 & 35 & 90 & 9 \\
\hline 5 & 2 & 3 & 5000 & 48 & 110 & 11 \\
\hline 6 & 2 & 4 & 5000 & 66 & 140 & 14 \\
\hline
\end{tabular}

1 Does not fulfil requirements.

Two curves with input voltage as abscissas and output current as ordinates with circuit 1 and one curve with circuit 2 are shown in Fig. 2. The irregularity of the sloping part indicates that the input current is distorted slightly by the amplifier due to the grid current taken by the tubes. 


\section{Discussion}

Table 1 shows that in order to fulfil the conditions that the input current of 10 to 20 milliamperes will be amplified to $110^{-}$to 200 milliamperes, the minimum number of tubes that can be used is four tubes in circuit 1 and 5 tubes in circuit 2. Circuit ${ }^{\bar{\top}} 1$ should therefore be the more economical to use even though two separate filament batteries are required, because less tubes are required and the total current capacity of both batteries

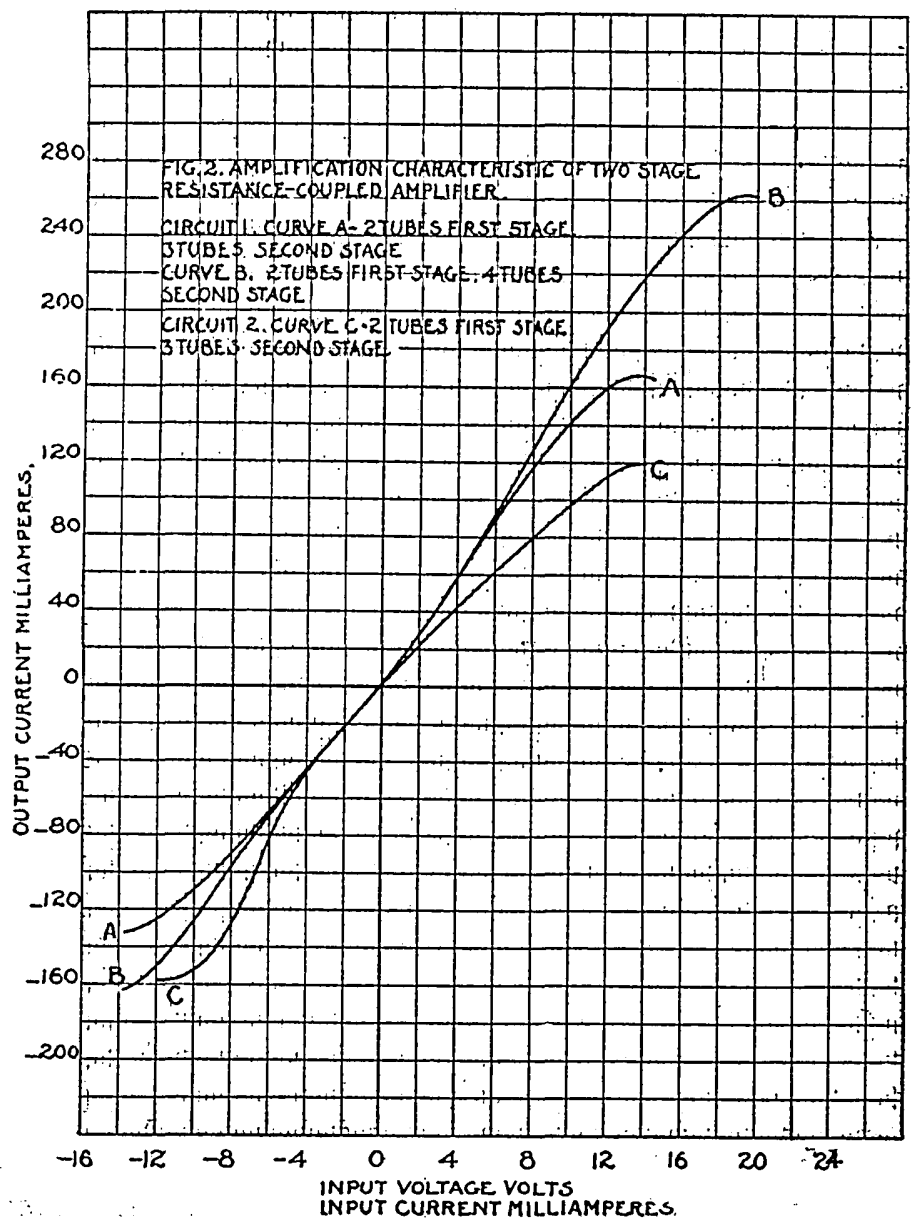

FIG. 2-Amplifier characteristics 
required to supply the filaments in circuit 1 is less than the capacity of the single battery required in circuit 2. Circuit 2 also requires an additional grid battery $\left(B_{6}\right.$, Fig. 1$)$ of 12 volts.

The difference in amplification of the two circuits is due to the manner of connecting the coupling resistance $\left(R_{2}\right.$, Fig. 1). In circuit 1 , the grid current of the second stage tubes acts as a regenerator, to increase the amplification for a given input current, while in circuit 2 the amplification is decreased by the grid current.

\section{Adjustments}

The necessary adjustments to put the amplifier in proper operating condition are:

(1) Filament Current: The filament current is adjusted by means of a series rheostat to 2.3 amperes per tube, or the filament voltage to 7.4 volts. With an 8 volt storage battery and low resistance leads no external resistance is necessary.

(2) Output Current: With no input current flowing, the resistance $R_{3}$ of Fig. 1 , is adjusted until the output current is reduced to zero. The amplifier is now ready for operation.

If changes occur in the plate voltage supply or a tube is replaced, it may be necessary to readjust $R_{3}$ to reduce the output current to zero with zero input current.

\section{GeNERAL}

The plate supply $\left(B_{4}\right.$, of Fig. 1$)$ may be of any voltage between 200 and 250 volts. A 220 volt D. C. light or power line may be used provided that the voltage does not vary more than about 10 volts.

With a plate supply of less than 200 volts the amplification decreases rapidly, while voltages in excess of 250 overheat the tubes and make their operation unsteady.

The above tests were all made using $U V-202$ Radiotron 5-watt power tubes. Other tubes suitable for current amplification are Western Electric type $E$ tubes. With these tubes somewhat less amplification was obtained, and not as steady operation because of heating. 


\section{SUMMARY}

Two resistance-coupled amplifier circuits were constructed to amplify both a direct and alternating current of 10 milliamperes to 110 to 200 milliamperes magnitude, and tests made to determine the amplification for best adjustments of the circuit constants.

BuREaU of Standards,

Washington, D. C. 